\title{
5-YEAR EXPERIENCE OF MINIMALLY INVASIVE TREATMENT OF BAKER CYSTS IN CHILDREN IN CONDITIONS OF OUTPATIENT SURGERY CENTER
}

\section{Ilja Krestyashin ${ }^{\bowtie}$ (D), Vladimir Krestyashin $(\mathbb{D}$, Olga Zimina (10)}

Department of Pediatric Surgery, Russian Scientific Research University; Filatovskaja Hospital, Moscow

krest_xirurg@mail.ru

ABSTRACT - The article deals with a method of treatment of Baker cysts in children by evacuation of cysts content followed by an orthopedic treatment, which has been carried out over 5 years in conditions of an outpatient surgery center.

KEYW ORDS - Baker cysts in children, drainage, outpatient surgery center.

\section{INTRODUCTION}

In modern surgical practice, the relevance of approaches and tactics for treatment of cyst Bakers have not yet been identified and do not have a unique protocol.

There are various methods of conservative and operational treatment of this pathology, starting from compresses with medicinal herbs, ending with a complete simultaneous excision of the cyst cavity.

\section{MATERIAL AND METHODS}

360 children with Baker cysts aged 3 to 12 years underwent observation in our orthopedic center over the past 5 years. To each patient an ultrasound examination was assigned, education level identified as well as differential diagnostics with phlebectasis of the popliteal region was conducted. If the cyst is tense on palpation, its volume exceeds $10 \mathrm{~cm}^{3}$ or an obvious cosmetic defect patients were offered a drainage treatment aiming at evacuating the contents of the cyst through a Brown catheter with subsequent washing of the cavity with $2 \%$ novocaine solution and application back plaster casts with a stiffener for a period of 14 days with restrictive home regimen and subsequent wearing of the knee orthosis for a period of 1 month.

If after an ultrasound examination the cyst volume does not exceed $10 \mathrm{~cm}^{3}$, patients were prescribed a follow-up examination after 3 months with a control ultrasound checkup. In the event of a decrease in cyst volume, the patient remained under dynamic observation every 3 months, if the volume increased, we puncture treatment was performed according to the method described above.

Among 360 patients, puncture was performed in 76 children with a sex ratio: $67 \%$ boys, $33 \%$ girls.

After 3 months, increase in the cyst cavity was observed in 17 children.

Relapse in the near term $-5-7$ weeks after a puncture in 3 patients (due to violation of the restrictive regime). Relapse in remote periods $-2-3$ months after the puncture (resumption of active sports activities such as gymnastics, football, tennis) 5 children. Accordingly, to puncture treatment this algorithm of actions was employed only in $25.83 \%$ of cases.

\section{CONCLUSIONS}

The obtained results, in our opinion, determine this technique as a priority, enabling to avoid the operative intervention and the introduction of cytostatics in the paraarticular region.

\section{REFERENCES}

1. Popliteal cysts in paediatric patients: clinical characteristics and imaging features on ultrasound and MRI. Neubauer H, et al. Arthritis. 2011 doi: $10.1155 / 2011 / 751593$

2. Natural history of popliteal cysts in the pediatric population. Akagi R, et al. J Pediatr Orthop. 2013 AprMay doi: 10.1097/BPO.0b013e318281e9bf.

3. Baker's cyst in pediatric patients: Ultrasonographic characteristics. Alessi S, et al. J Ultrasound. 2012 doi: 10.1016/j.jus.2011.06.007

4. Diagnosis and Therapy of Popliteal Cyst. Jin-Yu Wang et al. Zhongguo Gu Shang. 2019 DOI:10.3969/j. issn.1003-0034.2019.02.018 